\title{
ARTICLE \\ Present Status of Aquatic Resource and Its Catch of Mogra River in Bangladesh
}

\section{Chakraborty, B. K. . $^{*}$ Verma A. K. ${ }^{2}$ Muniya, S. ${ }^{3}$}

1. Department of Fisheries, Bangladesh and Agricultural University, Bangladesh

2. Govt. P. G. college, Saidabad, Prayaagraj, India

3. Bangladesh Agricultural University, Bangladesh

\begin{tabular}{l} 
ARTICLE INFO \\
\hline Article history \\
Received: 15 August 2021 \\
Accepted: 8 September 2021 \\
Published Online: 20 September 2021 \\
\hline Keywords: \\
Aquatic fauna \\
Biodiversity \\
Rare \\
Critically endangered \\
Endangered \\
Illegal fishing \\
Over exploitation
\end{tabular}

\section{Introduction}

River ecosystems and biodiversity help in maintaining the ecological balance of the waterbody. There is a necessity of ecological balance for widespread biodiversity and the ecological balance is an indispensable need for human survival ${ }^{[1]}$. The biodiversity conservation and environmental ethics both are required for sustainable development and survival of aquatic flora and fauna because biodiversity is the foundation of human life ${ }^{[2]}$.

Biodiversity has become a major concern to the fisher-

\begin{abstract}
Bangladesh is very rich in aquatic fauna with a biodiversity. The present study, conducted during 2015 to 2019 , recorded a total number of 131 species (104 fish, 09 prawn, 01 snail, 04 crabs, and 13 turtles) belonging to 26 families were identified from the Mogra River and its flood plain. About ten types of fishing gears, different crafts, hook and line were found operative in the river. Increasing rates of using current jal (16.0-26.40\%) and Kapuri jal (11.0-16.70\%) were identified as detrimental gears destroying different species. The fish productivity was decreased dramatically from $170.63 \pm 10.81 \mathrm{mt}$ to $134.75 \pm 8.02 \mathrm{mt}$ with a decreasing percentage of 6.26 to $21.03 \%$ within five years. Three important aquatic species turtiles (Cyclemys oldhami, Melanocheelys trjuuga and Morenia petersi) became rare and 17 commercially important aquatic species were at the edge of extinction (critically endangered, CR). From the study, 67 species were recorded in the endangered (EN) category, 20 species vulnerable status (VU), 11 species lower risk (LR), 07 species Least concern (LC) and 04 Data deficient (DF). To save the existing aquatic species in the studied riverine ecosystem and ensure better livelihood of the fishes, a team of local management committee, similar to the Hilsa fisheries management technology is needed.
\end{abstract}

ies biologists against the backdrop of rapid decline in the natural population of fish and aquatic biota across all the continents of the world. Biodiversity encompasses genetic species, assemblage, ecosystem and land cape levels of biological organization with structural, compositional and functional components ${ }^{[3,4]}$. Though loss of aquatic species has been occurring rapidly, the aquatic organisms have received comparatively little attention from conservation biologists ${ }^{[5]}$. A rich diversity of fish species is important to the ecology and sustainable productivity of the flood plains ${ }^{[6]}$. The resource of aquatic fauna in Bangladesh are

*Corresponding Author:

Chakraborty, B. K.,

Department of Fisheries, Bangladesh and Agricultural University, Bangladesh;

Email: bborty@gmail.com 
under severe threat due to over-exploitation and environmental degradation, which includes human interventions through construction of flood control embankments, drainage structures and sluice gates, conversion of inundated land to cropland thereby reducing water area and indiscriminate use of pesticides. Pollution from domestic, industrial and agrochemicals wastes has resulted in extinction of a considerable amount of aquatic biota in some stretches of the open water system ${ }^{[7,8]}$.

The upper region of the Mogra River is connected with Bisnai River and Kangshow River. The riverine flows across the Atpara and Modon Upazilla of Netrokona district from northern to southern Tharail and Itna Upazilla of Kishorgong District, before joining the Surma River. The water flow is continuous in the river. During monsoon, the water flow comes down from the upper region of Kangshow River and water flow does not confine within the banks. As a result, it causes floods in some area of Atpara and Modan Upazilla every year.

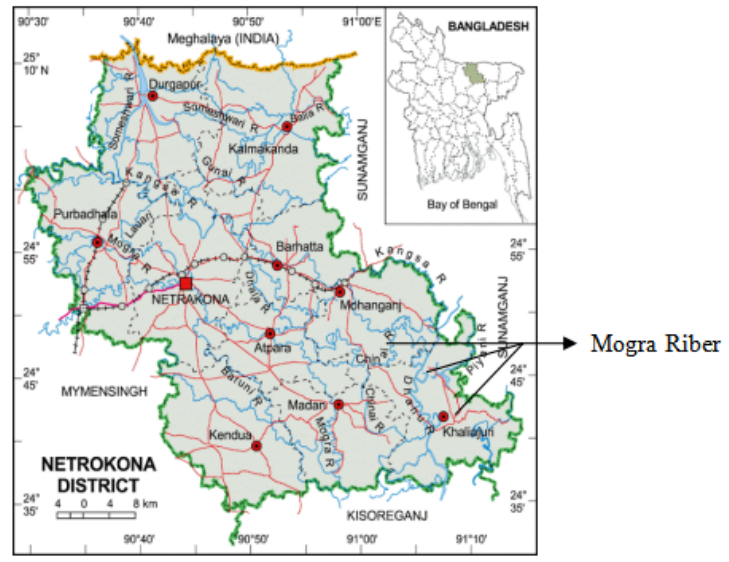

Figure 1. Location of Mogra River in the Netrokona district, Bangladesh.

Once upon a time, Mogra River was an abundance of native wild fishes, shrimp, crabs and reptiles. Due to over-exploitation and various ecological changes of the Mogra River, important fish species, and reptiles disappeared. Now this river is under great stress and its existence is endangered because of the changing aquatic ecosystems. The upper stream of the riverine system is siltated, which reduces the rate of water flow and causes habitat degradation. Like other floodplains, the feeding and breeding grounds of fishes in and around the river have been reducing drastically from various human created obstacles. Indiscriminate destructive fishing practices, soil erosion, siltation, construction of flood control and drainage structures, and agro-chemicals and pesticide have caused havoc to the aquatic biodiversity in Bangladesh.

\section{Methodology}

\section{Experimental design}

Mogra River was studied during 2015-2019 with particular emphasis on soil and water quality, biological productivity and status exploitation of the fishery resources. The river comprises an average length of 20-22 km long course. For the purpose of the study the river course was divided into upper and lower regions based on soil structure, water quality and fishing activities. The river courses of Atpara to Nazirgonj constitute the upper region while the Nazirgonj to Madon constituted the lower region, where in the Mogra River joins with the Surma River.

\section{Study of water quality parameters}

The bamboo made meter scale was used to measure water depth. Water temperature $\left({ }^{\circ} \mathrm{C}\right)$ was recorded using a Celsius thermometer and transparency $(\mathrm{cm})$ was measured using a Secchi disc (20 cm diameter). Dissolved oxygen $(\mathrm{mg} / \mathrm{l})$ and $\mathrm{pH}$ were measured directly using a digital electronic oxygen meter (YSI, Model 58, USA) and an electronic pH meter (Jenway, Model 3020, UK). Alkalinity was determined following the titrimetric method.

\section{Sampling of fish}

The investigation was conducted from 2015-2019 and was sampled simultaneously for winter (mid November to mid February), pre monsoon (mid February to April), monsoon (May to August) and post monsoon (September to mid November) for assessment of fish abundance and availability.

\section{Data collection}

The study was based on both primary and secondary data, comprehensive literature review and extracts of local knowledge and information. An organized sampling program spread over a reasonably long time is needed to get a true picture of the catch and composition. This study, being a rapid survey, gives only a broad picture of the stock of fishes, prawn, crabs and turtiles that could be obtained through market survey (Brojer Bazar, Nazirgong Bazar, Teligati Bazar, Madon sadar Bazar) and interaction with fishers in the riverside and even in the river and secondary data were collected from the Department of Fisheries (DoF) and the internet. The number of six codes (CR, E, EN, VU, LR, LC and DD) of IUCN was followed to categorize the coservation of status of fishes recorded from the river and to compare the trend among Shannon index value of different years ${ }^{[9]}$. 


\section{Shannon Diversity Index}

$$
\mathrm{H}=\sum_{\mathrm{i}=1}^{\mathrm{s}}-\left(\mathrm{P}_{\mathrm{i}} * \ln \mathrm{P}_{\mathrm{i}}\right)
$$

Where:

$\mathrm{H}=$ the Shannon diversity index, $\mathrm{P}_{\mathrm{i}}=$ fraction of the entire population made up of species $i, S=$ numbers of species encountered, $\sum=$ sum from species 1 to species $\mathrm{S}$.

Note: The power to which the base e $(\mathrm{e}=2.718281828$. $)$ must be raised to obtain a number is called the natural logarithm (ln) of the number.

\section{Analysis of experimental data}

The data were analyzed through one way ANOVA using MSTAT followed by Duncan's Multiple Range Test to find out whether any significant difference existed among the different means ${ }^{[10]}$.

\section{Results and Discussion}

\section{Morphometry and hydrodynamics of experim- ental river}

Generally, there are three main sources of water input into the river ecosystem viz. overspill from the higher river channel, surface flow and regeneration. Water flows were resolved by both rainfall and flooded water from the Meghaloya's hilly range, India. In upper region, this river is connected with Khongsa and Bisnai River. Flooding of the river originated from the Kangshow and Bisnai River. Surface run-off and increased in river height due to inflow of rainwater (flood) from the upper stretch, cause inundation of floodplains. The more water gain or exchange of water took place during southwest monsoon when floodplains were flooded. The early flood phase (April to June) occurred in the early monsoon when the water level in basin was relatively low. The water level in the floodplain rises and falls depending on the water level in adjacent rivers. The deep flood phase (June to September) began when the water level in the river, causing deep flooding in the four unions of Atpara and Madon Upazillas. Floodwater in flood plains began started receding in the post-monsoon season (October to December). The water loss by various means caused shrinkage of the effective water area and lowering of depth in the river which is very similar to the study of Chakraborty et al. ${ }^{[11]}$.

\section{Physical characteristics}

Soil texture of the Mogra River bed varied from sandy to loam sand. Soil texture of upper river bed was having $90.80 \pm 6.02$ sandy, $7.30 \pm 2.43$ loam sand and $1.9 \pm 1.72 \%$ clay. The dominance of sand (58.30 \pm 5.18$)$ was also recorded in the lower region of the river (Table 1).

Table 1. Physical features of sediment of the Mogra River.

\begin{tabular}{|c|c|c|c|}
\hline \multirow{2}{*}{ Location } & \multicolumn{3}{|c|}{ Soil texture of the river bed (\%) } \\
\cline { 2 - 4 } & Sandy & Loam sand & Clay \\
\hline Upper region & $40.20 \pm 4.32$ & $43.60 \pm 5.03$ & $17.4 \pm 3.22$ \\
\hline Lower region & $38.30 \pm 4.18$ & $42.10 \pm 4.06$ & $19.60 \pm 3.54$ \\
\hline
\end{tabular}

The waterw depth of the Mogra River exhibited a decreasing with an average value of $3.55 \pm 0.643 .41 \pm 0.55$, $3.321 \pm 0.5843 .207 \pm 0.44$ and $3.01 \pm 0.41 \mathrm{~m}$ during the study period (Figure 2). The highest depth of the river was recorded in the year 2015 and the lowest depth was found in the year 2019 and the equation of the trend line was $y=$ $-0.128 x+3.684\left(R^{2}=0.981\right)$. The alarming trend of decrease in water depth (Figure 2) was majorly due to rapid siltation ${ }^{[11]}$. The observed values of the value of the physico-chemical parameters of the river water are given in Table 2 . The temperature, transparency, $\mathrm{pH}$, dissolve oxygen and alkalinity of water were found to be more or less in the desired range. The variations in mean water temperature of the river were not statistically significant $(\mathrm{P}>0.05)$. Water temperature of the river showed an increasing trend in monsoon and post monsoon and decreasing trend in winter which was similar observation of Mathew ${ }^{[12]}$. Mean Secchi disk transparency differed significantly $(\mathrm{P}<0.05)$, during the study period. Higher values were recorded during post monsoon and summer months due to reduced flow and relatively stable conditions of water as observed by others ${ }^{[13]}$. The $\mathrm{pH}$ of the studied river did not differ significantly $(\mathrm{P}>0.05)$. Transparency was consistently higher in upper region and in the deeper portion of the river. A significant rise in $\mathrm{pH}$ during pre-monsoon and a drop in winter was noted in the river. The mean dissolved oxygen (DO) did not differ significantly ( $\mathrm{P}>0.05$ ). The $\mathrm{pH}$ and oxygen values of the river agreed more or less similar with the findings of APHA ${ }^{[14]}$ and Boyd ${ }^{[15]}$. Water alkalinity levels were recorded medium to high as reported by Clesceri et al. ${ }^{[16]}$. It differed significantly $(\mathrm{P}<0.05)$ with time. Lowest value of alkalinity was recorded in the in the winter during 2015. 
Table 2. Physico-chemical parameters of experimental Mogra River.

\begin{tabular}{|c|c|c|c|c|c|}
\hline \multirow{2}{*}{ Parameters } & \multicolumn{5}{|c|}{ Years } \\
\cline { 2 - 6 } & 2015 & 2016 & 2017 & 2018 & 2019 \\
\hline \multirow{2}{*}{ Temperature $\left({ }^{\circ} \mathrm{C}\right)$} & $25.74 \pm 5.01$ & $26.17 \pm 6.12$ & $26.48 \pm 6.08$ & $26.88 \pm 6.26$ & $26.14 \pm 5.88$ \\
& $(14.04-32.20)$ & $(13.73-32.40)$ & $(14.11-31.85)$ & $(14.00-32.01)$ & $(14.15-32.08)$ \\
\hline \multirow{2}{*}{ Transparency $(\mathrm{cm})$} & $40.04 \pm 6.24^{\mathrm{d}}$ & $50.38 \pm 7.02^{\mathrm{a}}$ & $44.55 \pm 6.41^{\mathrm{c}}$ & $37.19 \pm 6.88^{\mathrm{e}}$ & $47.23 \pm 6.74^{\mathrm{b}}$ \\
& $(30.10-50.16)$ & $(32.22-58.14)$ & $(28.15-50.30)$ & $(27.55-50.25)$ & $(29.55-55.22)$ \\
\hline \multirow{2}{*}{$\mathrm{pH}$} & $7.05 \pm 2.04$ & $7.66 \pm 2.22$ & $8.05 \pm 2.03$ & $7.77 \pm 1.88$ & $8.08 \pm 2.01$ \\
& $(6.90-8.86)$ & $(6.80-8.88)$ & $(6.85-9.07)$ & $(6.90-8.88)$ & $(6.75-8.90)$ \\
\hline Dissolved oxygen & $6.95 \pm 1.84$ & $8.84 \pm 1.88$ & $7.70 \pm 1.99$ & $7.22 \pm 1.72$ & $7.09 \pm 1.96$ \\
$(\mathrm{mg} / \mathrm{L})$ & $(4.18-8.04)$ & $(4.55-9.05)$ & $(5.44-8.66)$ & $(5.41-8.05)$ & $(5.04-8.48)$ \\
\hline \multirow{2}{*}{ Alkalinity (mg/L) } & $142.02 \pm 10.04^{\mathrm{a}}$ & $120.66 \pm 7.22^{\mathrm{e}}$ & $126.18 \pm 7.05^{\mathrm{d}}$ & $131.52 \pm 8.07^{\mathrm{c}}$ & $136.38 \pm 7.04^{\mathrm{b}}$ \\
& $(111.22-151.05)$ & $(110.88-135.02)$ & $(107.22-138.15)$ & $(110.40-140.32)$ & $(111.16-144.55)$ \\
\hline
\end{tabular}

Figures with different superscripts in the same row varied significantly $(P>0.05)$.

Figures in the parenthesis indicate the range.

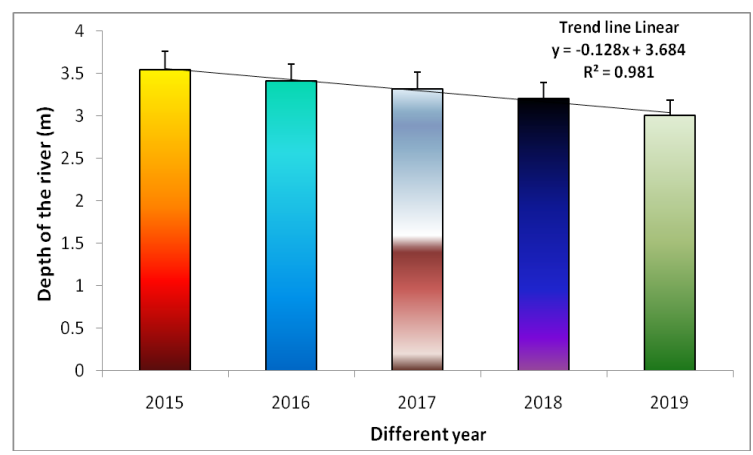

Figure 2. Water depth of the Mogra River between the year 2015 and 2019

\section{Capture method}

The fishers used wooden boats as a major craft. They used seine net (Bar jal and Komor jal), Thela jal, Dharma jal, Bua jal, Lift net, Cast net, Current jal and various types of fish Trap, Hook and Line according to season and availability of different species of fishes. Wide variability in fish traps (vair, dugair, ghuni and pholo etc.) and hook and line (barshi, fulkuichi, Jhupi aikra etc.) were used to capture different groups of aquatic lives.

Figure 3 shows a remarkable yearly increase in fishing effort by using illegal fishing gear like gill net (Current jal) and Bar jal (kaperi jal) in the total catch. The percentage of catch from Current jal were $14.00 \%, 16.20 \%$, $19.80 \%, 22.00 \%$ and $26.20 \%$; and Bar jal (kaperi jal) $12.00 \%, 13.70 \%, 14.50 \%, 15.10 \%$ and $16.50 \%$; and Hook and line $10.00,10.50,11.00,11.60$ and $11.70 \%$ in the year 2015, 2016, 2017, 2018 and 2019, respectively. Significant difference in catch $(P<0.05)$ by Current jal and Bar jal (kaperi jal) and Hook and line were identified. The contribution of catch by Komor jal were $13.00 \%, 12.80 \%$, $12.50 \%, 12.30 \%$ and $11.70 \%$ in the years 2015,2016 , 2017, 2018 and 2019, respectively. 
jal, Cast net, Traps, and line and Hooks to catch fishes. Fishermen also operated kata fishing by seine net (Bar jal and Komor jal) in winter and spring. The catch is consisted of knife fish, major carp and minor carp, small fish, cat fish and small cat fish, eels, prawn, crabs and reptiles (Table 3 and Figure 4). The assessment of yearly total catch from the river was around $170.63 \pm 10.81 \mathrm{mt}, 159.93 \pm 9.80$ $\mathrm{mt} 150.98 \pm 10.66 \mathrm{mt}, 143.16 \pm 9.80$ ton and $134.75 \pm 8.02$ mt during 2015, 2016, 2017, 2018 and 2019, respectivly (Figure 5). The catch trend line was exponential type and the equation was $\mathrm{y}=180.3 \mathrm{e}^{-0.05 x}\left(\mathrm{R}^{2}=0.999\right)$.

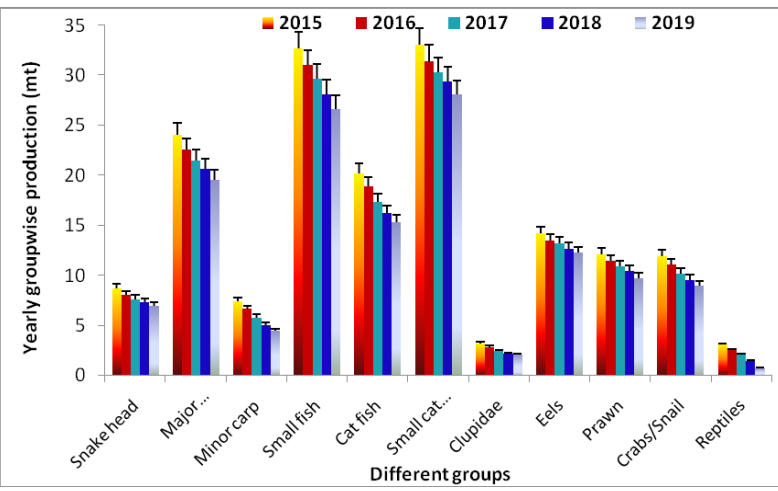

Figure 4. The production of different groups of aquatic lives in the Mogra River in the year 2015 to 2019.

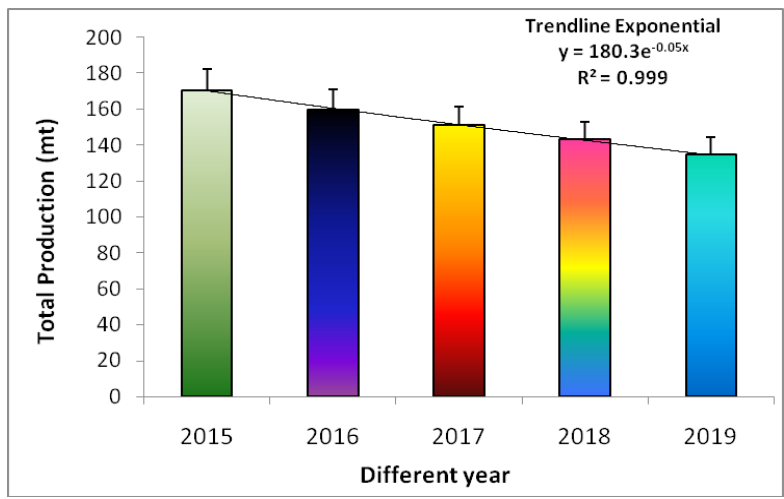

Figure 5. Decreasing trend in the total production of aquatic lives in the Mogra River during 2015 to 2019.

The fish catch showed a decrease percentage at the rate of $6.26 \%, 11.52 \%, 16.10 \%$ and $21.03 \%$ of catch in the years 2015-2016, 2016-2017, 2017-2018 and 20182019 , with respect to the catch of 2015 (Figure 6) and which exhibited a linear trend line and the equation was $\mathrm{y}=4.889 \mathrm{x}+1.5 .5\left(\mathrm{R}^{2}=0.999\right)$. A decrease trend in production from the river was clearly pronounced within the study period of five years which was similar to the study of Chakraborty and Mirza ${ }^{[19,20]}$ and Moyle and Leidy ${ }^{[21]}$. Although the production of all the recorded groups decreased during the study, it was pronounced more for reptiles.

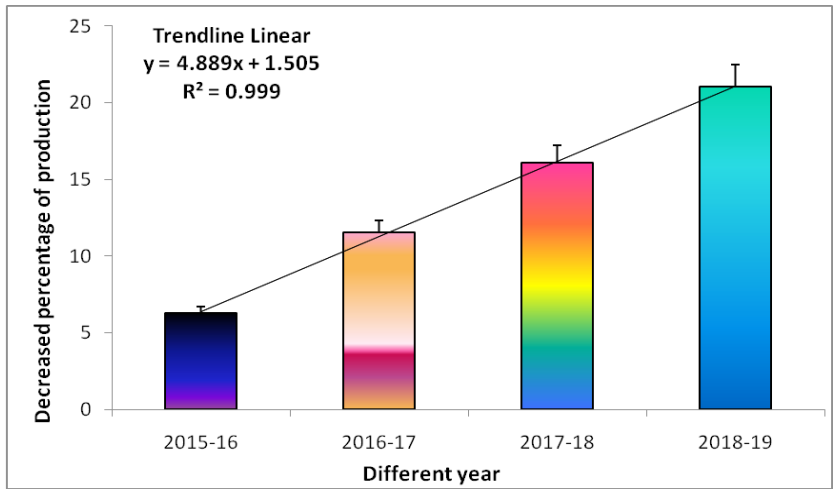

Figure 6. Decreasing percentage of total production of aquatic lives in the Mogra River during 2015 to 2019.

Table 3 and Figure 7 exhibited the conservation status of the 131 aquatic wild animals of the Mogra River and identified as E- 04 (3\%), CR-17 (12\%), EN-67 (51\%), VU-20 (15\%), LR-11 (9\%), LC-7 (9\%) and DD-06 (4\%), respectively.

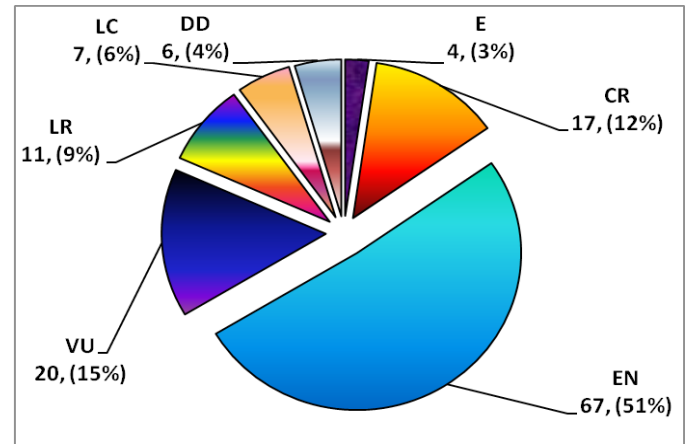

Figure 7. Conservation status of the recorded aquatic species in the Mogra River.

Status code: E- Extinct, CR- Critically Endangered, EN- Endangered, VU- Vulnerable, LR- Lower risk, LCNot threatened DD=Data deficient (As per IUCN ${ }^{[22]}$ ).

The total catch in different years differed significantly $(P<0.05)$. Commercial important Pata Kachim, Cyclemys oldhami, Kali Kachhap, Melanocheelys trjunga and Bengal Eyed Turtile, Morenia petersi were rarely found in the years 2015 to 2017 in the river. However these species were not recorded during 2019. Channa marulius, Puntius sarana, Barilius tileo, Sicamugil casoasia, Rohtee cotio, Bagarius yarrellii, Mystus seenghala, Bagarius yarrellii, Chaca chaca, Rama chandramara, Sisor rabdophorus, Pseudolaguvia muricata, Pseudolaguvia inornata and reptiles (Indotestudo elongata, Batagur baska, Geoclemys hamiltonii and Pangshura tecta (17 species) were reported as critically endangered and facing an extremely high risk of extinction in the river system (Table 3). According to IUCN ${ }^{[23]}$, in Bangladesh, about 56 freshwater fish species are critically or somewhat endangered. Due to Over 
Sustainable Marine Structures | Volume 03 | Issue 02 | July 2021

Table 3. Status and distribution of Mogra River of northern Bangladesh.

\begin{tabular}{|c|c|c|c|c|c|c|c|c|c|}
\hline \multirow{3}{*}{$\begin{array}{l}\text { SL } \\
\text { No }\end{array}$} & \multirow{3}{*}{ Group/ Family } & \multirow{3}{*}{ Local name } & \multirow{3}{*}{ Scientific name } & \multicolumn{5}{|c|}{ Production (mt) } & \multirow{3}{*}{ Status } \\
\hline & & & & \multicolumn{5}{|c|}{ Someswari River } & \\
\hline & & & & 2015 & 2016 & 2017 & 2018 & 2019 & \\
\hline \multicolumn{10}{|c|}{ Snake headed } \\
\hline 1 & Notopteridae & Chitol & Notopterus chitala & $\begin{array}{c}0.70 \\
\pm 0.09 \\
\end{array}$ & $\begin{array}{c}0.65 \\
\pm 0.05 \\
\end{array}$ & $\begin{array}{c}0.59 \\
\pm 0.05 \\
\end{array}$ & $\begin{array}{c}0.54 \\
\pm 0.03 \\
\end{array}$ & $\begin{array}{c}0.50 \\
\pm 0.02 \\
\end{array}$ & $\mathrm{EN}$ \\
\hline 2 & Notopteridae & Foli & Notopterus notopterus & $\begin{array}{c}0.50 \\
\pm 0.04\end{array}$ & $\begin{array}{c}0.44 \\
\pm 0.03\end{array}$ & $\begin{array}{c}0.37 \\
\pm 0.02\end{array}$ & $\begin{array}{c}0.33 \\
\pm 0.01 \\
\end{array}$ & $\begin{array}{c}0.30 \\
\pm 0.01\end{array}$ & $\mathrm{EN}$ \\
\hline 3 & Belonidae & Kakila & Xenentodon cancila & $\begin{array}{c}1.90 \\
\pm 0.44 \\
\end{array}$ & $\begin{array}{c}1.70 \\
\pm 0.40 \\
\end{array}$ & $\begin{array}{c}1.55 \\
\pm 0.41 \\
\end{array}$ & $\begin{array}{c}1.38 \\
\pm 1.10 \\
\end{array}$ & $\begin{array}{c}1.26 \\
\pm 0.07 \\
\end{array}$ & LR \\
\hline 4 & Channidae & Gojar & Channa marulius & $\begin{array}{c}0.60 \\
\pm 0.20\end{array}$ & $\begin{array}{c}0.53 \\
\pm 0.09\end{array}$ & $\begin{array}{c}0.50 \\
\pm 0.07\end{array}$ & $\begin{array}{c}0.48 \\
\pm 0.05\end{array}$ & $\begin{array}{c}0.44 \\
\pm 0.03\end{array}$ & $\mathrm{CR}$ \\
\hline 5 & Channidae & Soal & Channa striata & $\begin{array}{c}1.03 \\
\pm 0.51 \\
\end{array}$ & $\begin{array}{c}1.00 \\
\pm 0.10\end{array}$ & $\begin{array}{c}0.97 \\
\pm 0.08\end{array}$ & $\begin{array}{c}0.98 \\
\pm 0.09\end{array}$ & $\begin{array}{c}0.92 \\
\pm 0.06\end{array}$ & $\mathrm{EN}$ \\
\hline 7 & Channidae & Taki & Channa punctata & $\begin{array}{c}2.08 \\
\pm 0.60 \\
\end{array}$ & $\begin{array}{c}2.00 \\
\pm 0.55 \\
\end{array}$ & $\begin{array}{c}1.98 \\
\pm 0.50 \\
\end{array}$ & $\begin{array}{r}1.95 \\
\pm 0.44 \\
\end{array}$ & $\begin{array}{c}1.90 \\
\pm 0.42 \\
\end{array}$ & LR \\
\hline \multicolumn{4}{|c|}{ SubTotal } & $\begin{array}{c}8.89 \\
\pm 0.69 \\
\end{array}$ & $\begin{array}{c}8.02 \\
\pm 0.68 \\
\end{array}$ & $\begin{array}{c}7.64 \\
\pm 0.64 \\
\end{array}$ & $\begin{array}{c}7.32 \\
\pm 0.63 \\
\end{array}$ & $\begin{array}{c}6.94 \\
\pm 0.62 \\
\end{array}$ & \\
\hline \multicolumn{10}{|c|}{ Major carps } \\
\hline 1 & Cyprinidae & Catla & Catla catla & $\begin{array}{c}1.98 \\
\pm 0.80 \\
\end{array}$ & $\begin{array}{c}1.84 \\
\pm 0.65 \\
\end{array}$ & $\begin{array}{c}1.80 \\
\pm 0.60 \\
\end{array}$ & $\begin{array}{c}1.73 \\
\pm 0.54 \\
\end{array}$ & $\begin{array}{c}1.65 \\
\pm 0.50 \\
\end{array}$ & $\mathrm{EN}$ \\
\hline 2 & Cyprinidae & Rui & Labeo rohita & $\begin{array}{c}3.01 \\
\pm 0.30 \\
\end{array}$ & $\begin{array}{c}2.88 \\
\pm 0.30 \\
\end{array}$ & $\begin{array}{c}2.81 \\
\pm 0.30 \\
\end{array}$ & $\begin{array}{c}2.71 \\
\pm 0.27 \\
\end{array}$ & $\begin{array}{c}2.60 \\
\pm 0.21 \\
\end{array}$ & $\mathrm{EN}$ \\
\hline 3 & Cyprinidae & Mrigal & Cirrhinus mrigala & $\begin{array}{c}3.04 \\
\pm 0.26 \\
\end{array}$ & $\begin{array}{c}3.0 \\
\pm 0.24 \\
\end{array}$ & $\begin{array}{c}2.97 \\
\pm 0.22 \\
\end{array}$ & $\begin{array}{c}2.90 \\
\pm 0.21 \\
\end{array}$ & $\begin{array}{c}2.78 \\
\pm 0.20 \\
\end{array}$ & $\mathrm{EN}$ \\
\hline 4 & Cyprinidae & Kalbaus & Labeo calbasu & $\begin{array}{c}2.3 \\
\pm 0.19 \\
\end{array}$ & $\begin{array}{c}2.25 \\
\pm 0.18 \\
\end{array}$ & $\begin{array}{c}2.21 \\
\pm 0.17 \\
\end{array}$ & $\begin{array}{c}2.16 \\
\pm 0.10 \\
\end{array}$ & $\begin{array}{c}2.07 \\
\pm 0.10 \\
\end{array}$ & $\mathrm{EN}$ \\
\hline 6 & Cyprinidae & Reba & Cirrhinus reba & $\begin{array}{c}1.80 \\
\pm 0.11 \\
\end{array}$ & $\begin{array}{c}1.60 \\
\pm 0.10 \\
\end{array}$ & $\begin{array}{c}1.40 \\
\pm 0.09 \\
\end{array}$ & $\begin{array}{c}1.20 \\
\pm 0.08 \\
\end{array}$ & $\begin{array}{c}1.10 \\
\pm 0.06 \\
\end{array}$ & $\mathrm{EN}$ \\
\hline 7 & Cyprinidae & Common carp & Cyprinus carpio & $\begin{array}{c}5.50 \\
\pm 1.84 \\
\end{array}$ & $\begin{array}{c}5.20 \\
\pm 1.70 \\
\end{array}$ & $\begin{array}{c}5.00 \\
\pm 1.22 \\
\end{array}$ & $\begin{array}{c}4.80 \\
\pm 1.10 \\
\end{array}$ & $\begin{array}{c}4.50 \\
\pm 1.00 \\
\end{array}$ & VU \\
\hline 8 & Cyprinidae & Grass carp & Ctenopharyngodon idella & $\begin{array}{c}3.60 \\
\pm 1.24 \\
\end{array}$ & $\begin{array}{c}3.30 \\
\pm 1.11 \\
\end{array}$ & $\begin{array}{c}3.00 \\
\pm 1.01 \\
\end{array}$ & $\begin{array}{c}2.95 \\
\pm 1.00 \\
\end{array}$ & $\begin{array}{c}2.88 \\
\pm 0.98 \\
\end{array}$ & VU \\
\hline \multicolumn{4}{|c|}{ Sub-Total } & $24.03 \pm 1.17$ & $22.57 \pm 1.12$ & $21.49 \pm 1.09$ & $\begin{array}{l}20.65 \\
\pm 1.08 \\
\end{array}$ & $\begin{array}{r}19.55 \\
\pm 1.03 \\
\end{array}$ & \\
\hline \multicolumn{10}{|c|}{ Minor carp } \\
\hline 1 & Cyprinidae & Along & Bengala elanga & $\begin{array}{c}1.20 \\
\pm 0.07 \\
\end{array}$ & $\begin{array}{c}1.10 \\
\pm 0.06 \\
\end{array}$ & $\begin{array}{c}0.94 \\
\pm 0.04 \\
\end{array}$ & $\begin{array}{c}0.82 \\
\pm 0.03 \\
\end{array}$ & $\begin{array}{c}0.69 \\
\pm 0.01 \\
\end{array}$ & $\mathrm{VU}$ \\
\hline 2 & Cyprinidae & Bhangna bata & Labeo bata & $\begin{array}{c}1.10 \\
\pm 0.06 \\
\end{array}$ & $\begin{array}{c}1.00 \\
\pm 0.05 \\
\end{array}$ & $\begin{array}{c}0.80 \\
\pm 0.05 \\
\end{array}$ & $\begin{array}{c}0.65 \\
\pm 0.04 \\
\end{array}$ & $\begin{array}{c}0.50 \\
\pm 0.02 \\
\end{array}$ & $\mathrm{EN}$ \\
\hline 3 & Cyprinidae & Ghora muikha & Labeo pangusia & $\begin{array}{c}1.82 \\
\pm 0.05 \\
\end{array}$ & $\begin{array}{c}1.80 \\
\pm 0.05 \\
\end{array}$ & $\begin{array}{c}1.70 \\
\pm 0.04 \\
\end{array}$ & $\begin{array}{c}1.60 \\
\pm 0.03 \\
\end{array}$ & $\begin{array}{c}1.50 \\
\pm 0.03 \\
\end{array}$ & $\mathrm{EN}$ \\
\hline 4 & Cyprinidae & Jarua/Utti & Chagunius chagunio & $\begin{array}{c}0.87 \\
\pm 0.06 \\
\end{array}$ & $\begin{array}{c}0.70 \\
\pm 0.05 \\
\end{array}$ & $\begin{array}{c}0.62 \\
\pm 0.03 \\
\end{array}$ & $\begin{array}{c}0.48 \\
\pm 0.03 \\
\end{array}$ & $\begin{array}{c}0.38 \\
\pm 0.01 \\
\end{array}$ & $\mathrm{EN}$ \\
\hline 5 & Cyprinidae & Puda & Puntius sarana & $\begin{array}{c}0.58 \\
\pm 0.04 \\
\end{array}$ & $\begin{array}{c}0.45 \\
\pm 0.03 \\
\end{array}$ & $\begin{array}{c}0.22 \\
\pm 0.02 \\
\end{array}$ & $\begin{array}{c}0.12 \\
\pm 0.01 \\
\end{array}$ & $\begin{array}{c}0.01 \\
\pm 0.01 \\
\end{array}$ & $\mathrm{CR}$ \\
\hline 6 & Cyprinidae & Tila koksa & Barilius tileo & $\begin{array}{c}1.01 \\
\pm 0.08 \\
\end{array}$ & $\begin{array}{c}0.90 \\
\pm 0.05 \\
\end{array}$ & $\begin{array}{c}0.88 \\
\pm 0.04 \\
\end{array}$ & $\begin{array}{c}0.84 \\
\pm 0.03 \\
\end{array}$ & $\begin{array}{c}.81 \\
\pm 0.01 \\
\end{array}$ & $\mathrm{EN}$ \\
\hline 7 & Cyprinidae & Bhol & Raimass bola & $\begin{array}{c}0.86 \\
\pm 0.05 \\
\end{array}$ & $\begin{array}{c}0.72 \\
\pm 0.04 \\
\end{array}$ & $\begin{array}{c}0.64 \\
\pm 0.04 \\
\end{array}$ & $\begin{array}{c}0.55 \\
\pm 003 \\
\end{array}$ & $\begin{array}{c}0.54 \\
\pm 0.01 \\
\end{array}$ & $\mathrm{EN}$ \\
\hline \multicolumn{4}{|c|}{ Sub-Total } & $\begin{array}{c}7.74 \\
\pm 0.39 \\
\end{array}$ & $\begin{array}{c}6.67 \\
\pm 0.43 \\
\end{array}$ & $\begin{array}{c}5.80 \\
\pm 0.45 \\
\end{array}$ & $\begin{array}{c}5.06 \\
\pm 0.47 \\
\end{array}$ & $\begin{array}{c}4.43 \\
\pm 0.46 \\
\end{array}$ & \\
\hline \multicolumn{10}{|c|}{ Small fish } \\
\hline 1 & Cyprinidae & Mola & Amblypharyngodon mola & $\begin{array}{c}1.88 \\
\pm 0.07 \\
\end{array}$ & $\begin{array}{c}1.77 \\
\pm 0.06 \\
\end{array}$ & $\begin{array}{c}1.64 \\
\pm 0.05 \\
\end{array}$ & $\begin{array}{c}1.53 \\
\pm 0.05 \\
\end{array}$ & $\begin{array}{c}1.45 \\
\pm 0.03 \\
\end{array}$ & $\mathrm{EN}$ \\
\hline
\end{tabular}


Sustainable Marine Structures | Volume 03 | Issue 02 | July 2021

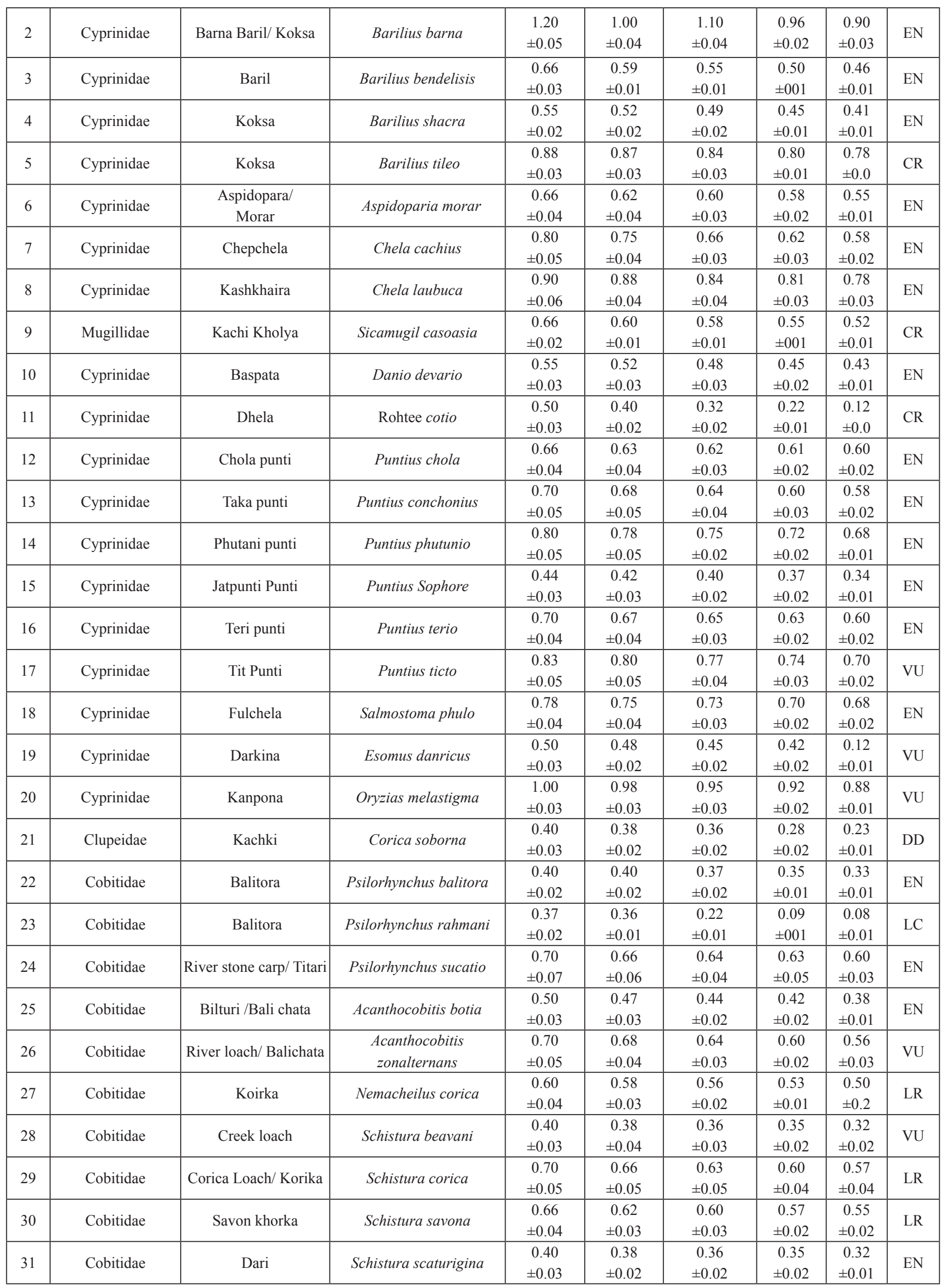


Sustainable Marine Structures | Volume 03 | Issue 02 | July 2021

\begin{tabular}{|c|c|c|c|c|c|c|c|c|c|}
\hline 32 & Cobitidae & $\begin{array}{c}\text { Bengal loach / Bou } \\
\text { mach }\end{array}$ & Botia dario & $\begin{array}{c}0.60 \\
\pm 0.05\end{array}$ & $\begin{array}{c}0.55 \\
\pm 0.04\end{array}$ & $\begin{array}{c}0.53 \\
\pm 0.04\end{array}$ & $\begin{array}{c}0.51 \\
\pm 0.02\end{array}$ & $\begin{array}{c}0.48 \\
\pm 0.02\end{array}$ & VU \\
\hline 33 & Cobitidae & Hora loach & Botia dayi & $\begin{array}{c}0.60 \\
\pm 0.05\end{array}$ & $\begin{array}{c}0.58 \\
\pm 0.04\end{array}$ & $\begin{array}{c}0.56 \\
\pm 0.03\end{array}$ & $\begin{array}{c}0.53 \\
\pm 0.03\end{array}$ & $\begin{array}{c}0.50 \\
\pm 0.01\end{array}$ & EN \\
\hline 34 & Cobitidae & Loach/ Puiya & $\begin{array}{c}\text { Lepidocephalichthys } \\
\text { goalparensis }\end{array}$ & $\begin{array}{c}0.90 \\
\pm 0.05\end{array}$ & $\begin{array}{c}0.88 \\
\pm 0.04\end{array}$ & $\begin{array}{c}0.85 \\
\pm 0.02\end{array}$ & $\begin{array}{c}0.83 \\
\pm 0.02\end{array}$ & $\begin{array}{c}0.81 \\
\pm 0.02\end{array}$ & $\mathrm{EN}$ \\
\hline 35 & Cobitidae & Goalpara loach & $\begin{array}{c}\text { Neoeucirrhichthys } \\
\text { maydelli }\end{array}$ & $\begin{array}{c}0.55 \\
\pm 0.04\end{array}$ & $\begin{array}{c}0.52 \\
\pm 0.04\end{array}$ & $\begin{array}{c}0.50 \\
\pm 0.03\end{array}$ & $\begin{array}{c}0.48 \\
\pm 0.02 \\
\end{array}$ & $\begin{array}{c}0.45 \\
\pm 0.01\end{array}$ & $\mathrm{EN}$ \\
\hline 36 & Cobitidae & $\begin{array}{c}\text { Gonga loach/ } \\
\text { Poia/ Ghar poia }\end{array}$ & Somileptes gongota & $\begin{array}{c}0.60 \\
\pm 0.05\end{array}$ & $\begin{array}{c}0.58 \\
\pm 0.05\end{array}$ & $\begin{array}{c}0.55 \\
\pm 0.04\end{array}$ & $\begin{array}{c}0.53 \\
\pm 0.03\end{array}$ & $\begin{array}{c}0.50 \\
\pm 0.02\end{array}$ & VU \\
\hline 37 & Cobitidae & Rani & Botia lohachata & $\begin{array}{c}0.44 \\
\pm 0.04\end{array}$ & $\begin{array}{c}0.41 \\
\pm 0.04\end{array}$ & $\begin{array}{c}0.38 \\
\pm 0.03\end{array}$ & $\begin{array}{c}0.36 \\
\pm 0.03\end{array}$ & $\begin{array}{c}0.33 \\
\pm 0.01\end{array}$ & LR \\
\hline 38 & Cobitidae & Rani & $\begin{array}{c}\text { Lepidocephalichthys } \\
\text { annandalei }\end{array}$ & $\begin{array}{c}0.55 \\
\pm 0.03\end{array}$ & $\begin{array}{c}0.53 \\
\pm 0.03\end{array}$ & $\begin{array}{c}0.52 \\
\pm 0.02 \\
\end{array}$ & $\begin{array}{c}0.50 \\
\pm 0.02 \\
\end{array}$ & $\begin{array}{c}0.47 \\
\pm 0.02\end{array}$ & $\mathrm{EN}$ \\
\hline 39 & Cobitidae & Balichata & Nemachilus botia & $\begin{array}{c}0.77 \\
\pm 0.04\end{array}$ & $\begin{array}{c}0.74 \\
\pm 0.04\end{array}$ & $\begin{array}{c}0.73 \\
\pm 0.02\end{array}$ & $\begin{array}{c}0.71 \\
\pm 0.03\end{array}$ & $\begin{array}{c}0.68 \\
\pm 0.03\end{array}$ & $\mathrm{EN}$ \\
\hline 40 & Centropomidae & Chanda & Chanda nama & $\begin{array}{c}1.50 \\
\pm 0.08\end{array}$ & $\begin{array}{c}1.47 \\
\pm 0.05\end{array}$ & $\begin{array}{c}1.44 \\
\pm 0.04\end{array}$ & $\begin{array}{c}1.36 \\
\pm 004\end{array}$ & $\begin{array}{c}1.28 \\
\pm 0.03\end{array}$ & LC \\
\hline 41 & Centropomidae & Chanda & Pseudambasis bacuculis & $\begin{array}{c}1.20 \\
\pm 0.08 \\
\end{array}$ & $\begin{array}{c}1.16 \\
\pm 0.06 \\
\end{array}$ & $\begin{array}{c}1.15 \\
\pm 0.05 \\
\end{array}$ & $\begin{array}{c}1.13 \\
\pm 0.04 \\
\end{array}$ & $\begin{array}{c}1.08 \\
\pm 0.04 \\
\end{array}$ & $\mathrm{EN}$ \\
\hline 42 & Centropomidae & Ranga chanda & Pseudambasis ranga & $\begin{array}{c}0.80 \\
\pm 0.05\end{array}$ & $\begin{array}{c}0.74 \\
\pm 0.04\end{array}$ & $\begin{array}{c}0.70 \\
\pm 0.03\end{array}$ & $\begin{array}{c}0.68 \\
\pm 0.03\end{array}$ & $\begin{array}{c}0.66 \\
\pm 0.02\end{array}$ & $\mathrm{LC}$ \\
\hline 43 & Gobiidae & Baila & Glossogobus giuris & $\begin{array}{c}1.20 \\
\pm 0.07\end{array}$ & $\begin{array}{c}1.10 \\
\pm 0.06\end{array}$ & $\begin{array}{c}1.00 \\
\pm 0.05\end{array}$ & $\begin{array}{c}0.98 \\
\pm 0.04\end{array}$ & $\begin{array}{c}0.94 \\
\pm 0.04\end{array}$ & $\mathrm{DD}$ \\
\hline 44. & Tetradontidae & Potka & Tetradon cutcutia & $\begin{array}{c}1.50 \\
\pm 0.08\end{array}$ & $\begin{array}{c}1.44 \\
\pm 0.07\end{array}$ & $\begin{array}{c}1.44 \\
\pm 0.06\end{array}$ & $\begin{array}{c}1.36 \\
\pm 0.04\end{array}$ & $\begin{array}{c}1.32 \\
\pm 0.04\end{array}$ & $\mathrm{EN}$ \\
\hline \multicolumn{4}{|c|}{ Sub-Total } & $\begin{array}{l}32.72 \\
\pm 0.32\end{array}$ & $29.53 \pm 0.29$ & $29.63 \pm 0.30$ & $\begin{array}{r}28.14 \\
\pm 0.29 \\
\end{array}$ & $\begin{array}{r}26.63 \\
\pm 0.28 \\
\end{array}$ & \\
\hline \multicolumn{10}{|c|}{ Cat fish } \\
\hline 2 & Bagridae & Guizza & Mystus seenghala & $\begin{array}{c}3.00 \\
\pm 0.20\end{array}$ & $\begin{array}{c}2.89 \\
\pm 0.17\end{array}$ & $\begin{array}{c}2.75 \\
\pm 0.14\end{array}$ & $\begin{array}{c}2.66 \\
\pm 0.11\end{array}$ & $\begin{array}{c}2.55 \\
\pm 0.11\end{array}$ & CR \\
\hline 3 & Schilbeidae & Shilong & Silonia silondia & $\begin{array}{c}1.00 \\
\pm 0.09\end{array}$ & $\begin{array}{c}0.97 \\
\pm 0.08\end{array}$ & $\begin{array}{c}0.93 \\
\pm 0.09\end{array}$ & $\begin{array}{c}0.90 \\
\pm 0.07\end{array}$ & $\begin{array}{c}0.88 \\
\pm 0.08\end{array}$ & $\mathrm{EN}$ \\
\hline 4 & Siluridae & Boal & Wallago attu & $\begin{array}{c}5.03 \\
\pm 1.84 \\
\end{array}$ & $\begin{array}{r}4.90 \\
\pm 1.71 \\
\end{array}$ & $\begin{array}{r}4.70 \\
\pm 1.81 \\
\end{array}$ & $\begin{array}{c}4.40 \\
\pm 1.70 \\
\end{array}$ & $\begin{array}{l}41.00 \\
\pm 1.40 \\
\end{array}$ & LR \\
\hline 5 & Bagridae & Baghair & Bagarius yarrellii & $\begin{array}{c}2.08 \\
\pm 0.80\end{array}$ & $\begin{array}{c}1.66 \\
\pm 0.70\end{array}$ & $\begin{array}{l}1.20 \\
\pm 0.7 \\
\end{array}$ & $\begin{array}{c}1.11 \\
\pm 0.68\end{array}$ & $\begin{array}{c}1.00 \\
\pm 0.65\end{array}$ & CR \\
\hline 6 & Chacidae & Cheka & Chaca chaca & $\begin{array}{c}1.50 \\
\pm 0.10\end{array}$ & $\begin{array}{c}1.30 \\
\pm 0.09\end{array}$ & $\begin{array}{c}1.00 \\
\pm 0.08\end{array}$ & $\begin{array}{c}0.96 \\
\pm 0.08\end{array}$ & $\begin{array}{c}0.90 \\
\pm 0.05\end{array}$ & CR \\
\hline 7 & Bagridae & Gangmagur & Mystus menoda & $\begin{array}{c}2.85 \\
\pm 0.90 \\
\end{array}$ & $\begin{array}{c}2.55 \\
\pm 0.80 \\
\end{array}$ & $\begin{array}{c}2.33 \\
\pm 0.78 \\
\end{array}$ & $\begin{array}{c}2.00 \\
\pm 0.74 \\
\end{array}$ & $\begin{array}{c}1.88 \\
\pm 0.60 \\
\end{array}$ & $\mathrm{EN}$ \\
\hline 8 & Bagridae & Rita & Rita rita & $\begin{array}{c}2.55 \\
\pm 0.81\end{array}$ & $\begin{array}{c}2.50 \\
\pm 0.70\end{array}$ & $\begin{array}{c}2.44 \\
\pm 0.70\end{array}$ & $\begin{array}{c}2.20 \\
\pm 0.60\end{array}$ & $\begin{array}{c}2.09 \\
\pm 0.50\end{array}$ & $\mathrm{EN}$ \\
\hline Sub t & & & & $20.21 \pm 1.21$ & $18.87 \pm 1.22$ & $17.35 \pm 1.24$ & $\begin{array}{l}16.21 \\
\pm 1.15\end{array}$ & $\begin{array}{r}15.30 \\
\pm 1.08 \\
\end{array}$ & \\
\hline \multicolumn{10}{|c|}{ Small cat fish } \\
\hline 1 & Bagridae & Gulsa & Mystus cavasius & $\begin{array}{c}2.20 \\
\pm 0.12 \\
\end{array}$ & $\begin{array}{c}2.10 \\
\pm 0.11 \\
\end{array}$ & $\begin{array}{c}2.08 \\
\pm 0.08 \\
\end{array}$ & $\begin{array}{c}2.03 \\
\pm 0.07 \\
\end{array}$ & $\begin{array}{c}1.90 \\
\pm 0.06 \\
\end{array}$ & $\mathrm{EN}$ \\
\hline 2 & Bagridae & Tengra & Mystus vitttus & $\begin{array}{c}2.70 \\
\pm 0.11\end{array}$ & $\begin{array}{c}2.60 \\
\pm 0.11\end{array}$ & $\begin{array}{c}2.50 \\
\pm 0.10\end{array}$ & $\begin{array}{c}2.45 \\
\pm 0.10\end{array}$ & $\begin{array}{c}2.40 \\
\pm 0.08\end{array}$ & EN \\
\hline 3 & Bagridae & Bujuri & Mystus tengra & $\begin{array}{c}2.70 \\
\pm 0.11\end{array}$ & $\begin{array}{c}2.66 \\
\pm 0.11\end{array}$ & $\begin{array}{c}2.60 \\
\pm 0.08\end{array}$ & $\begin{array}{c}2.55 \\
\pm 0.07\end{array}$ & $\begin{array}{c}2.51 \\
\pm 0.06\end{array}$ & VU \\
\hline 4 & Bagridae & $\begin{array}{c}\text { Gura Tengra/ Futki } \\
\text { bujuri }\end{array}$ & Rama chandramara & $\begin{array}{c}0.70 \\
\pm 0.06\end{array}$ & $\begin{array}{c}0.60 \\
\pm 0.04\end{array}$ & $\begin{array}{c}0.50 \\
\pm 0.03\end{array}$ & $\begin{array}{c}0.48 \\
\pm 0.03\end{array}$ & $\begin{array}{c}0.39 \\
\pm .0 .02 \\
\end{array}$ & CR \\
\hline 5 & Bagridae & $\begin{array}{c}\text { Menoda catfish } \\
\text { /Arwari }\end{array}$ & Hemibagrus menoda & $\begin{array}{c}0.80 \\
\pm 0.07\end{array}$ & $\begin{array}{c}0.77 \\
\pm 0.05\end{array}$ & $\begin{array}{c}0.75 \\
\pm 0.05\end{array}$ & $\begin{array}{c}0.73 \\
\pm 0.04\end{array}$ & $\begin{array}{c}0.70 \\
\pm 0.02\end{array}$ & $\mathrm{EN}$ \\
\hline
\end{tabular}


Sustainable Marine Structures | Volume 03 | Issue 02 | July 2021

\begin{tabular}{|c|c|c|c|c|c|c|c|c|c|}
\hline 7 & Bagridae & $\begin{array}{c}\text { Day's mystus/ } \\
\text { Tengra }\end{array}$ & Mystus bleekeri & $\begin{array}{c}0.75 \\
\pm 0.07 \\
\end{array}$ & $\begin{array}{c}0.74 \\
\pm 0.05\end{array}$ & $\begin{array}{c}0.72 \\
\pm 0.05 \\
\end{array}$ & $\begin{array}{c}0.70 \\
\pm 0.04 \\
\end{array}$ & $\begin{array}{c}0.68 \\
\pm 0.02 \\
\end{array}$ & EN \\
\hline 8 & Schilbeidae & Kajuli & Ailia coila & $\begin{array}{c}0.90 \\
\pm 0.08\end{array}$ & $\begin{array}{c}0.86 \\
\pm 0.07\end{array}$ & $\begin{array}{c}0.84 \\
\pm 0.06\end{array}$ & $\begin{array}{c}0.81 \\
\pm 0.05\end{array}$ & $\begin{array}{c}0.79 \\
\pm 0.04\end{array}$ & EN \\
\hline 9 & Siluridae & Kani Pabda & Ompok bimaculatus & $\begin{array}{c}1.58 \\
\pm 0.08\end{array}$ & $\begin{array}{c}1.50 \\
\pm 0.07\end{array}$ & $\begin{array}{c}1.48 \\
\pm 0.06\end{array}$ & $\begin{array}{c}1.41 \\
\pm 0.06\end{array}$ & $\begin{array}{c}1.37 \\
\pm 0.05\end{array}$ & EN \\
\hline 10 & Siluridae & Madhu Pabda & Ompok pabda & $\begin{array}{c}1.77 \\
\pm 0.09\end{array}$ & $\begin{array}{c}1.60 \\
\pm 0.08\end{array}$ & $\begin{array}{c}1.55 \\
\pm 0.07\end{array}$ & $\begin{array}{c}1.52 \\
\pm 0.06\end{array}$ & $\begin{array}{c}1.48 \\
\pm 0.05\end{array}$ & VU \\
\hline 11 & Siluridae & Ompok pabda & Ompok pabo & $\begin{array}{c}1.20 \\
\pm 0.06\end{array}$ & $\begin{array}{c}1.17 \\
\pm 0.05\end{array}$ & $\begin{array}{c}1.14 \\
\pm 0.06\end{array}$ & $\begin{array}{c}1.10 \\
\pm 0.04\end{array}$ & $\begin{array}{c}1.00 \\
\pm 0.04\end{array}$ & EN \\
\hline 12 & Schilbeidae & Gharua & Clupisoma garua & $\begin{array}{c}1.07 \\
\pm 0.08\end{array}$ & $\begin{array}{c}0.96 \\
\pm 0.06\end{array}$ & $\begin{array}{c}0.94 \\
\pm 0.07\end{array}$ & $\begin{array}{c}0.88 \\
\pm 0.07\end{array}$ & $\begin{array}{c}0.80 \\
\pm 0.05\end{array}$ & EN \\
\hline 13 & Schilbeidae & Muri Bacha & Clupisoma murias & $\begin{array}{c}1.40 \\
\pm 0.05\end{array}$ & $\begin{array}{c}1.30 \\
\pm 0.05\end{array}$ & $\begin{array}{c}1.26 \\
\pm 0.05\end{array}$ & $\begin{array}{l}1.20 \\
0.04 \\
\end{array}$ & $\begin{array}{c}1.14 \\
\pm 0.03\end{array}$ & $\mathrm{EN}$ \\
\hline 14 & Schilbeidae & Batasi & $\begin{array}{c}\text { Pseudeutropius } \\
\text { atherinoides }\end{array}$ & $\begin{array}{c}1.00 \\
\pm 0.05\end{array}$ & $\begin{array}{c}0.97 \\
\pm 0.05\end{array}$ & $\begin{array}{c}0.95 \\
\pm 0.03\end{array}$ & $\begin{array}{c}0.92 \\
\pm 0.02\end{array}$ & $\begin{array}{c}0.90 \\
\pm .0 .02\end{array}$ & $\mathrm{VU}$ \\
\hline 15 & Schilbeidae & Bacha & Eutropiichthys vacha & $\begin{array}{c}0.90 \\
\pm 0.04 \\
\end{array}$ & $\begin{array}{c}0.88 \\
\pm 0.05\end{array}$ & $\begin{array}{c}0.85 \\
\pm 0.04 \\
\end{array}$ & $\begin{array}{c}0.83 \\
\pm 0.04 \\
\end{array}$ & $\begin{array}{c}0.80 \\
\pm 0.02 \\
\end{array}$ & $\mathrm{EN}$ \\
\hline 16 & Sisoridae & Kutakanti & Hara hara & $\begin{array}{c}0.60 \\
\pm 0.04\end{array}$ & $\begin{array}{c}0.55 \\
\pm 0.04 \\
\end{array}$ & $\begin{array}{c}0.49 \\
\pm 0.04 \\
\end{array}$ & $\begin{array}{c}0.45 \\
\pm 0.04 \\
\end{array}$ & $\begin{array}{c}0.44 \\
\pm 0.04\end{array}$ & LR \\
\hline 17 & Sisoridae & Kutakanti & Hara jerdoni & $\begin{array}{c}1.10 \\
\pm 0.07\end{array}$ & $\begin{array}{c}1.04 \\
\pm 0.06\end{array}$ & $\begin{array}{c}1.00 \\
\pm 0.06\end{array}$ & $\begin{array}{c}0.99 \\
\pm 0.05\end{array}$ & $\begin{array}{c}0.96 \\
\pm 0.04\end{array}$ & EN \\
\hline 18 & Sisoridae & Gang tengra & Nangra nangra & $\begin{array}{c}0.90 \\
\pm 0.04 \\
\end{array}$ & $\begin{array}{c}0.88 \\
\pm 0.03 \\
\end{array}$ & $\begin{array}{c}0.85 \\
\pm 0.04 \\
\end{array}$ & $\begin{array}{l}0.82 \\
0.04 \\
\end{array}$ & $\begin{array}{c}0.79 \\
\pm 0.03 \\
\end{array}$ & VU \\
\hline 19 & Sisoridae & Chenua & Sisor rabdophorus & $\begin{array}{c}0.35 \\
\pm 0.02 \\
\end{array}$ & $\begin{array}{c}0.30 \\
\pm 0.02 \\
\end{array}$ & $\begin{array}{c}0.25 \\
\pm 0.03\end{array}$ & $\begin{array}{c}0.16 \\
\pm 0.02 \\
\end{array}$ & $\begin{array}{r}0.08 \\
\pm .0 .02 \\
\end{array}$ & CR \\
\hline 20 & Sisoridae & $\begin{array}{c}\text { Conta catfish/ Kuta } \\
\text { kanti }\end{array}$ & Conta conta & $\begin{array}{c}1.24 \\
\pm 0.07 \\
\end{array}$ & $\begin{array}{c}1.20 \\
\pm 0.06 \\
\end{array}$ & $\begin{array}{c}1.15 \\
\pm 0.05 \\
\end{array}$ & $\begin{array}{c}1.11 \\
\pm 0.04 \\
\end{array}$ & $\begin{array}{c}1.05 \\
\pm 0.02 \\
\end{array}$ & DD \\
\hline 21 & Sisoridae & Kutakanti & Erethistes pusillus & $\begin{array}{c}1.00 \\
\pm 0.02 \\
\end{array}$ & $\begin{array}{c}0.97 \\
\pm 0.02 \\
\end{array}$ & $\begin{array}{c}0.93 \\
\pm 0.01 \\
\end{array}$ & $\begin{array}{c}0.12 \\
\pm 0.01 \\
\end{array}$ & $\begin{array}{c}0.10 \\
\pm .0 .01 \\
\end{array}$ & VU \\
\hline 23 & Sisoridae & Chanua & Pseudolaguvia inornata & $\begin{array}{c}1.44 \\
\pm 0.09\end{array}$ & $\begin{array}{c}1.33 \\
\pm 0.08 \\
\end{array}$ & $\begin{array}{c}1.32 \\
\pm 0.06\end{array}$ & $\begin{array}{c}1.32 \\
\pm 0.05\end{array}$ & $\begin{array}{c}1.29 \\
\pm 0.05 \\
\end{array}$ & CR \\
\hline 24 & Clariidae & Cat fish/ Magur & Clarias batrachus & $\begin{array}{c}0.50 \\
\pm 0.03\end{array}$ & $\begin{array}{c}0.47 \\
\pm 0.03 \\
\end{array}$ & $\begin{array}{c}0.45 \\
\pm 0.03 \\
\end{array}$ & $\begin{array}{c}0.43 \\
\pm 0.02 \\
\end{array}$ & $\begin{array}{r}0.40 \\
\pm .0 .02 \\
\end{array}$ & $\mathrm{VU}$ \\
\hline 25 & Heteropneustidae & $\begin{array}{l}\text { Stinging catfish/ } \\
\text { Shingi }\end{array}$ & Heteropneustes fossilis & $\begin{array}{c}1.44 \\
\pm 0.07 \\
\end{array}$ & $\begin{array}{c}1.38 \\
\pm 0.05 \\
\end{array}$ & $\begin{array}{c}1.32 \\
\pm 0.05 \\
\end{array}$ & $\begin{array}{c}1.30 \\
\pm 0.04 \\
\end{array}$ & $\begin{array}{c}1.26 \\
\pm 0.02 \\
\end{array}$ & $\mathrm{LC}$ \\
\hline 26 & Chacidae & Cheka & Chaca chaca & $\begin{array}{c}1.70 \\
\pm 0.10 \\
\end{array}$ & $\begin{array}{c}1.60 \\
\pm 0.09 \\
\end{array}$ & $\begin{array}{c}1.15 \\
\pm 0.08 \\
\end{array}$ & $\begin{array}{c}1.47 \\
\pm 0.05 \\
\end{array}$ & $\begin{array}{c}1.33 \\
\pm .0 .05 \\
\end{array}$ & LR \\
\hline 27 & Olyridae & Gagora catfish / Gobi & Arius gagora & $\begin{array}{c}1.48 \\
\pm 0.07\end{array}$ & $\begin{array}{c}1.40 \\
\pm 0.06\end{array}$ & $\begin{array}{c}1.35 \\
\pm 0.05\end{array}$ & $\begin{array}{c}1.32 \\
\pm 0.04 \\
\end{array}$ & $\begin{array}{c}1.27 \\
\pm 0.02\end{array}$ & $\mathrm{EN}$ \\
\hline \multicolumn{4}{|c|}{ Sub-total } & $33.03 \pm 0.60$ & $31.45 \pm 0.58$ & $30.31 \pm 0.57$ & $\begin{array}{l}29.41 \\
\pm 0.57\end{array}$ & $\begin{array}{l}28.09 \\
\pm 0.56\end{array}$ & \\
\hline \multicolumn{10}{|c|}{ Clupidae } \\
\hline 1 & Clupidae & Chapila & Gadusia chapra & $\begin{array}{c}1.80 \\
\pm 0.08 \\
\end{array}$ & $\begin{array}{c}1.50 \\
\pm 0.07\end{array}$ & $\begin{array}{c}1.12 \\
\pm 0.06\end{array}$ & $\begin{array}{c}1.00 \\
\pm 0.05 \\
\end{array}$ & $\begin{array}{c}1.00 \\
\pm 0.04\end{array}$ & $\mathrm{EN}$ \\
\hline 2 & Clupidae & Hilsa & Tenualosa ilisha & $\begin{array}{c}0.98 \\
\pm 0.08 \\
\end{array}$ & $\begin{array}{c}0.95 \\
\pm 0.06\end{array}$ & $\begin{array}{c}0.90 \\
\pm 0.04\end{array}$ & $\begin{array}{c}0.85 \\
\pm 0.02\end{array}$ & $\begin{array}{c}0.82 \\
\pm 0.01 \\
\end{array}$ & $\mathrm{EN}$ \\
\hline 3 & Clupidae & $\begin{array}{c}\text { Gizzard shad/ } \\
\text { Chapila }\end{array}$ & Gonialosa manmina & $\begin{array}{c}0.44 \\
\pm 0.08\end{array}$ & $\begin{array}{c}0.38 \\
\pm 0.06\end{array}$ & $\begin{array}{c}0.34 \\
\pm 0.04 \\
\end{array}$ & $\begin{array}{c}0.31 \\
\pm 0.02 \\
\end{array}$ & $\begin{array}{c}0.28 \\
\pm 0.01 \\
\end{array}$ & $\mathrm{EN}$ \\
\hline \multicolumn{4}{|c|}{ Subtotal } & $\begin{array}{c}3.22 \\
\pm 0.68 \\
\end{array}$ & $\begin{array}{c}2.83 \\
\pm 0.56 \\
\end{array}$ & $\begin{array}{c}2.44 \\
\pm 0.44 \\
\end{array}$ & $\begin{array}{c}2.16 \\
\pm 0.36 \\
\end{array}$ & $\begin{array}{c}2.10 \\
\pm 0.38 \\
\end{array}$ & \\
\hline \multicolumn{10}{|l|}{ Eels } \\
\hline 1 & Mastacembeli-dae & Baim & Mastacembalus armatus & $\begin{array}{c}3.44 \\
\pm 0.14 \\
\end{array}$ & $\begin{array}{c}3.35 \\
\pm 0.11 \\
\end{array}$ & $\begin{array}{c}3.33 \\
\pm 0.09\end{array}$ & $\begin{array}{c}3.24 \\
\pm 0.08 \\
\end{array}$ & $\begin{array}{c}3.12 \\
\pm 0.07\end{array}$ & VU \\
\hline 2 & Synbranchidae & Kuicha & Monopterus cuchia & $\begin{array}{c}3.09 \\
\pm 0.10 \\
\end{array}$ & $\begin{array}{c}2.98 \\
\pm 0.10\end{array}$ & $\begin{array}{c}2.91 \\
\pm 0.09 \\
\end{array}$ & $\begin{array}{c}2.80 \\
\pm 0.08\end{array}$ & $\begin{array}{c}2.27 \\
\pm 0.08\end{array}$ & $\mathrm{EN}$ \\
\hline
\end{tabular}


Sustainable Marine Structures | Volume 03 | Issue 02 | July 2021

\begin{tabular}{|c|c|c|c|c|c|c|c|c|c|}
\hline 4 & Mastacembelidae & One-stripe spiny eel & Macrognathus aral & $\begin{array}{c}2.20 \\
\pm 0.12\end{array}$ & $\begin{array}{c}2.00 \\
\pm 0.11\end{array}$ & $\begin{array}{c}1.95 \\
\pm 0.09\end{array}$ & $\begin{array}{c}1.91 \\
\pm 0.08\end{array}$ & $\begin{array}{c}1.86 \\
\pm 0.07\end{array}$ & LR \\
\hline 5 & Mastacembelidae & $\begin{array}{l}\text { Barred spiny eel/ } \\
\text { Pankal baim }\end{array}$ & Macrognathus pancalus & $\begin{array}{c}2.55 \\
\pm 0.13\end{array}$ & $\begin{array}{c}2.30 \\
\pm 0.12\end{array}$ & $\begin{array}{c}2.22 \\
\pm 0.12\end{array}$ & $\begin{array}{c}2.12 \\
\pm 0.11\end{array}$ & $\begin{array}{c}2.04 \\
\pm 0.10\end{array}$ & EN \\
\hline \multicolumn{4}{|c|}{ Subtotal } & $\begin{array}{l}14.18 \\
\pm 0.48\end{array}$ & $\begin{array}{l}13.44 \\
\pm 0.54\end{array}$ & $\begin{array}{l}13.17 \\
\pm 0.55\end{array}$ & $\begin{array}{l}12.70 \\
\pm 0.53\end{array}$ & $\begin{array}{l}12.26 \\
\pm 0.51\end{array}$ & \\
\hline \multicolumn{10}{|c|}{ Prawn } \\
\hline 2 & Palaemonidae & Gura Isa & $\begin{array}{c}\text { Machrobrachium } \\
\text { biramanicus }\end{array}$ & $\begin{array}{c}2.50 \\
\pm 0.18\end{array}$ & $\begin{array}{c}2.42 \\
\pm 0.16\end{array}$ & $\begin{array}{c}2.35 \\
\pm 0.15\end{array}$ & $\begin{array}{c}2.28 \\
\pm 0.14\end{array}$ & $\begin{array}{c}2.20 \\
\pm 0.15\end{array}$ & DD \\
\hline 3 & Palaemonidae & Gul Isa & $\begin{array}{c}\text { Machrobrachium } \\
\text { malcolmsnii }\end{array}$ & $\begin{array}{c}1.61 \\
\pm 0.09\end{array}$ & $\begin{array}{c}1.44 \\
\pm 0.05\end{array}$ & $\begin{array}{c}1.32 \\
\pm 0.05\end{array}$ & $\begin{array}{c}1.25 \\
\pm 0.04\end{array}$ & $\begin{array}{c}1.18 \\
\pm 0.04\end{array}$ & VU \\
\hline 4 & Palaemonidae & Dimua icha & $\begin{array}{c}\text { Macrobrachium } \\
\text { villosimanus }\end{array}$ & $\begin{array}{c}1.90 \\
\pm 0.20\end{array}$ & $\begin{array}{c}1.80 \\
\pm 0.11\end{array}$ & $\begin{array}{c}1.71 \\
\pm 0.10\end{array}$ & $\begin{array}{c}1.64 \\
\pm 0.09\end{array}$ & $\begin{array}{c}1.57 \\
\pm 0.10\end{array}$ & $\mathrm{LC}$ \\
\hline 6 & Palaemonidae & $\begin{array}{c}\text { Kaira icha or beel } \\
\text { chingri. }\end{array}$ & Macrobrachium dayanum & $\begin{array}{c}0.71 \\
\pm 0.06\end{array}$ & $\begin{array}{c}0.66 \\
\pm 0.03\end{array}$ & $\begin{array}{c}0.60 \\
\pm 0.03\end{array}$ & $\begin{array}{c}0.60 \\
\pm 0.02\end{array}$ & $\begin{array}{c}0.54 \\
\pm 0.02 \\
\end{array}$ & LR \\
\hline 7 & Palaemonidae & Chikna chingri. & Macrobrachium idella & $\begin{array}{c}0.92 \\
\pm 0.02\end{array}$ & $\begin{array}{c}0.88 \\
\pm 0.02\end{array}$ & $\begin{array}{c}0.82 \\
\pm 0.01\end{array}$ & $\begin{array}{c}0.77 \\
\pm 0.01\end{array}$ & $\begin{array}{c}0.59 \\
\pm 0.01\end{array}$ & DD \\
\hline 8 & Palaemonidae & Icha & Macrobrachium kempi & $\begin{array}{c}0.87 \\
\pm 0.08\end{array}$ & $\begin{array}{c}0.82 \\
\pm 0.07\end{array}$ & $\begin{array}{c}0.78 \\
\pm 0.04\end{array}$ & $\begin{array}{c}0.75 \\
\pm 0.04\end{array}$ & $\begin{array}{c}0.72 \\
\pm 0.02\end{array}$ & VU \\
\hline 9 & Palaemonidae & chingri & Macrobrachium superbum & $\begin{array}{c}0.90 \\
\pm 0.06 \\
\end{array}$ & $\begin{array}{c}0.84 \\
\pm 0.04\end{array}$ & $\begin{array}{c}0.86 \\
\pm 0.03 \\
\end{array}$ & $\begin{array}{c}0.80 \\
\pm 0.02 \\
\end{array}$ & $\begin{array}{c}0.73 \\
\pm 0.02 \\
\end{array}$ & $\mathrm{LC}$ \\
\hline \multicolumn{4}{|c|}{ Sub-total: } & $\begin{array}{r}11.12 \\
\pm 0.63 \\
\end{array}$ & $\begin{array}{r}11.42 \\
\pm 0.62 \\
\end{array}$ & $\begin{array}{l}10.92 \\
\pm 0.60 \\
\end{array}$ & $\begin{array}{r}10.46 \\
\pm 0.57 \\
\end{array}$ & $\begin{array}{c}7.75 \\
\pm 0.56 \\
\end{array}$ & \\
\hline \multicolumn{10}{|c|}{ Crabs/Snail } \\
\hline 1 & Potamidae & Kakra & Sartoriana spinigera & $\begin{array}{c}2.77 \\
\pm 0.80 \\
\end{array}$ & $\begin{array}{c}2.73 \\
\pm 0.61 \\
\end{array}$ & $\begin{array}{c}2.54 \\
\pm 0.53\end{array}$ & $\begin{array}{c}2.46 \\
\pm 0.48 \\
\end{array}$ & $\begin{array}{c}240 \\
\pm 0.40 \\
\end{array}$ & DD \\
\hline 3 & Grapsidae & Kakra & Acanthopotamon martensi & $\begin{array}{c}2.48 \\
\pm 0.08 \\
\end{array}$ & $\begin{array}{c}2.33 \\
\pm 0.07\end{array}$ & $\begin{array}{c}2.12 \\
\pm 0.06 \\
\end{array}$ & $\begin{array}{c}2.00 \\
\pm 0.04\end{array}$ & $\begin{array}{c}1.90 \\
\pm 0.03 \\
\end{array}$ & VU \\
\hline 4 & Parathelphusidae & Kakra & Pyxidognathus fluviatilis & $\begin{array}{c}1.08 \\
\pm 0.03 \\
\end{array}$ & $\begin{array}{c}0.92 \\
\pm 0.03 \\
\end{array}$ & $\begin{array}{c}0.88 \\
\pm 0.02 \\
\end{array}$ & $\begin{array}{c}0.82 \\
\pm 0.02 \\
\end{array}$ & $\begin{array}{c}0.78 \\
\pm 0.01 \\
\end{array}$ & $\mathrm{LC}$ \\
\hline 5 & Parathelphusidae & Kakra & Austrotelphusa transversa & $\begin{array}{c}1.88 \\
\pm 0.04\end{array}$ & $\begin{array}{c}1.72 \\
\pm 0.05\end{array}$ & $\begin{array}{c}1.44 \\
\pm 0.04\end{array}$ & $\begin{array}{c}1.34 \\
\pm 0.03\end{array}$ & $\begin{array}{c}1.22 \\
\pm 0.01\end{array}$ & $\mathrm{EN}$ \\
\hline 6 & Unionidae & Bivalve & Lamellidens marginalis & $\begin{array}{c}1.12 \\
\pm 0.04 \\
\end{array}$ & $\begin{array}{c}1.00 \\
\pm 0.03 \\
\end{array}$ & $\begin{array}{c}0.90 \\
\pm 0.02 \\
\end{array}$ & $\begin{array}{c}0.85 \\
\pm 0.02 \\
\end{array}$ & $\begin{array}{c}0.79 \\
\pm 0.01 \\
\end{array}$ & VU \\
\hline \multicolumn{4}{|c|}{ Sub-total: } & $\begin{array}{r}11.93 \\
\pm 0.75 \\
\end{array}$ & $\begin{array}{r}11.10 \\
\pm 0.76\end{array}$ & $\begin{array}{l}10.21 \\
\pm 0.72 \\
\end{array}$ & $\begin{array}{c}9.57 \\
\pm 0.70 \\
\end{array}$ & $\begin{array}{c}8.97 \\
\pm 0.67 \\
\end{array}$ & \\
\hline \multicolumn{10}{|c|}{ Reptiles } \\
\hline 1 & Testudinidae & $\begin{array}{c}\text { Elongated Tortoise/ } \\
\text { Kachhap }\end{array}$ & Indotestudo elongata & $\begin{array}{c}0.29 \\
\pm 0.03 \\
\end{array}$ & $\begin{array}{c}0.22 \\
\pm 0.02 \\
\end{array}$ & $\begin{array}{c}0.19 \\
\pm 0.02 \\
\end{array}$ & $\begin{array}{c}0.13 \\
\pm 0.01 \\
\end{array}$ & $\begin{array}{c}0.09 \\
\pm 0.01 \\
\end{array}$ & $\mathrm{CR}$ \\
\hline 2 & Testudinidae & $\begin{array}{c}\text { Asian Giant Tortoise/ } \\
\text { Chila Kachhap }\end{array}$ & Manouria emys & $\begin{array}{c}0.35 \\
\pm 0.04 \\
\end{array}$ & $\begin{array}{c}0.32 \\
\pm 0.03 \\
\end{array}$ & $\begin{array}{c}0.21 \\
\pm 0.02 \\
\end{array}$ & $\begin{array}{c}0.18 \\
\pm 0.02 \\
\end{array}$ & $\begin{array}{c}0.11 \\
\pm 0.01 \\
\end{array}$ & $\mathrm{EN}$ \\
\hline 3 & Geoemydidae & $\begin{array}{c}\text { River Terrapin } \\
\text { /Bodo Kaitta }\end{array}$ & Batagur baska & $\begin{array}{c}0.16 \\
\pm 0.02 \\
\end{array}$ & $\begin{array}{c}0.13 \\
\pm 0.01\end{array}$ & $\begin{array}{c}0.09 \\
\pm 0.01\end{array}$ & $\begin{array}{c}0.05 \\
\pm 0.01\end{array}$ & $\begin{array}{c}0.01 \\
\pm 0.00\end{array}$ & CR \\
\hline 4 & Geoemydidae & $\begin{array}{c}\text { Painted Roofed } \\
\text { Turtile/Dhoor Kachim }\end{array}$ & Batagur dongoka & $\begin{array}{c}0.18 \\
\pm 0.02 \\
\end{array}$ & $\begin{array}{c}0.14 \\
\pm 0.02 \\
\end{array}$ & $\begin{array}{c}0.11 \\
\pm 0.01 \\
\end{array}$ & $\begin{array}{c}0.08 \\
\pm 0.01 \\
\end{array}$ & $\begin{array}{c}0.03 \\
\pm 0.07 \\
\end{array}$ & $\mathrm{EN}$ \\
\hline 5 & Geoemydidae & $\begin{array}{c}\text { Oldham,s Leaf Turtile/ } \\
\text { Pata Kachim }\end{array}$ & Cyclemys oldhami & $\begin{array}{c}0.26 \\
\pm 0.02 \\
\end{array}$ & $\begin{array}{c}0.20 \\
\pm 0.02\end{array}$ & $\begin{array}{c}0.14 \\
\pm 0.01\end{array}$ & $\begin{array}{c}010 \\
\pm 0.01\end{array}$ & $\begin{array}{c}0.00 \\
\pm 0.00\end{array}$ & $\mathrm{E}$ \\
\hline 6 & Geoemydidae & $\begin{array}{c}\text { SpottedTurtile/ } \\
\text { Kala Kachim }\end{array}$ & Geoclemys hamiltonii & $\begin{array}{c}0.19 \\
\pm 0.03\end{array}$ & $\begin{array}{c}0.14 \\
\pm 0.02\end{array}$ & $\begin{array}{c}0.12 \\
\pm 0.01 \\
\end{array}$ & $\begin{array}{c}0.10 \\
\pm 0.01 \\
\end{array}$ & $\begin{array}{c}0.07 \\
\pm 0.01\end{array}$ & $\mathrm{CR}$ \\
\hline 7 & Geoemydidae & $\begin{array}{c}\text { Brahminy River } \\
\text { Turtile/Kali Kaitta }\end{array}$ & Hardella thurjii & $\begin{array}{c}0.30 \\
\pm 0.11 \\
\end{array}$ & $\begin{array}{c}0.24 \\
\pm 0.05 \\
\end{array}$ & $\begin{array}{c}0.18 \\
\pm 0.06 \\
\end{array}$ & $\begin{array}{c}0.13 \\
\pm 0.02 \\
\end{array}$ & $\begin{array}{c}0.07 \\
\pm 0.01 \\
\end{array}$ & $\mathrm{EN}$ \\
\hline 8 & Geoemydidae & Shila Kachhap & $\begin{array}{c}\text { Melanocheelys } \\
\text { tricarinata }\end{array}$ & $\begin{array}{c}0.30 \\
\pm 0.02\end{array}$ & $\begin{array}{c}0.26 \\
\pm 0.01\end{array}$ & $\begin{array}{c}0.19 \\
\pm 0.01\end{array}$ & $\begin{array}{c}0.15 \\
\pm 0.01\end{array}$ & $\begin{array}{c}0.08 \\
\pm 0.00\end{array}$ & $\mathrm{EN}$ \\
\hline
\end{tabular}




\begin{tabular}{|c|c|c|c|c|c|c|c|c|c|}
\hline 9 & Geoemydidae & $\begin{array}{c}\text { Snail Eating Turtile/ } \\
\text { Kali Kachhap }\end{array}$ & $\begin{array}{c}\text { Melanocheelys } \\
\text { trjuuga }\end{array}$ & $\begin{array}{c}0.40 \\
\pm 0.02 \\
\end{array}$ & $\begin{array}{c}0.35 \\
\pm 0.02\end{array}$ & $\begin{array}{c}0.30 \\
\pm 0.01\end{array}$ & $\begin{array}{c}0.10 \\
\pm 0.00 \\
\end{array}$ & $\begin{array}{c}0.00 \\
\pm 0.00 \\
\end{array}$ & E \\
\hline 10 & Geoemydidae & Bengal Eyed Turtile & Morenia petersi & $\begin{array}{c}0.08 \\
\pm 0.01 \\
\end{array}$ & $\begin{array}{c}0.06 \\
\pm 0.01 \\
\end{array}$ & $\begin{array}{c}0.05 \\
\pm 0.01 \\
\end{array}$ & $\begin{array}{c}0.04 \\
\pm 0.00\end{array}$ & $\begin{array}{c}0.0 \\
\pm 0.00\end{array}$ & $\mathrm{E}$ \\
\hline 11 & Geoemydidae & $\begin{array}{c}\text { Indian Turtile/ Kori } \\
\text { Kaitta } \\
\end{array}$ & Pangshura tecta & $\begin{array}{c}0.13 \\
\pm 0.02 \\
\end{array}$ & $\begin{array}{c}0.10 \\
\pm 0.01\end{array}$ & $\begin{array}{c}0.10 \\
\pm 0.01 \\
\end{array}$ & $\begin{array}{c}0.09 \\
\pm 0.01 \\
\end{array}$ & $\begin{array}{c}0.07 \\
\pm 0.01 \\
\end{array}$ & $\mathrm{CR}$ \\
\hline 12 & Geoemydidae & $\begin{array}{c}\text { Tent Turtile/ } \\
\text { Majhari Kaitta } \\
\end{array}$ & Pangshura tentoria & $\begin{array}{c}0.07 \\
\pm 0.01 \\
\end{array}$ & $\begin{array}{c}0.06 \\
\pm 0.01 \\
\end{array}$ & $\begin{array}{c}0.06 \\
\pm 0.00 \\
\end{array}$ & $\begin{array}{c}0.05 \\
\pm 0.01 \\
\end{array}$ & $\begin{array}{c}0.04 \\
\pm 0.00 \\
\end{array}$ & EN \\
\hline 13 & Trionychidae & $\begin{array}{c}\text { Ganges Turtile/ Khalua } \\
\text { Kachim } \\
\end{array}$ & Aspideretes gangeticus & $\begin{array}{c}0.35 \\
\pm 0.03 \\
\end{array}$ & $\begin{array}{c}0.32 \\
\pm 0.02 \\
\end{array}$ & $\begin{array}{c}0.30 \\
\pm 0.03 \\
\end{array}$ & $\begin{array}{c}0.28 \\
\pm 0.02 \\
\end{array}$ & $\begin{array}{c}0.16 \\
\pm 0.01 \\
\end{array}$ & VU \\
\hline \multicolumn{4}{|c|}{ Sub-total } & $\begin{array}{c}3.06 \\
\pm 0.11 \\
\end{array}$ & $\begin{array}{c}2.54 \\
\pm 0.10 \\
\end{array}$ & $\begin{array}{r}2.04 \\
\pm 0.08 \\
\end{array}$ & $\begin{array}{c}1.48 \\
\pm 0.06 \\
\end{array}$ & $\begin{array}{c}0.73 \\
\pm 0.05 \\
\end{array}$ & \\
\hline & & & Total & $\begin{array}{c}170.63 \\
\pm 8.81 \\
\end{array}$ & $\begin{array}{c}159.93 \\
\pm 7.40 \\
\end{array}$ & $\begin{array}{c}150.98 \\
\pm 6.66 \\
\end{array}$ & $\begin{array}{c}143.16 \\
\pm 5.87 \\
\end{array}$ & $\begin{array}{l}134.75 \\
\pm 5.02 \\
\end{array}$ & \\
\hline
\end{tabular}

exploitation and various ecological changes in natural aquatic ecosystem of river and its floodplains, commercially important aquatic lives are in the verge of extinction which is in agreement with the findings of Sarker ${ }^{[24]}$.

The total catch data of the river exhibited a constant sharp decrease during 2015 and 2019. Some of the important native species were noted to be losing their presence. The capture of fishes, crab and reptiles in the river was recorded highest in 2015-16, but decreased considerably in 2017-2018 and the similar situation continued in 2018-2019. Small catfishes and small fishes are dominant groups caught from the river. The observation was similar to the findings of Chakraborty and Mirza ${ }^{[20]}$, Chakraborty ${ }^{[25]}$ and Chakraborty et al. ${ }^{[26,11]}$. As a result, commercially important three aquatic lives of river were recorded to be disappearing during this short 5 years experimental period.

A decreasing trend in catch of the river was clearly recorded within five years which was similar to the report of Chakraborty and Mirza ${ }^{[19]}$ and Moyle and Leidy ${ }^{[21]}$. A total of thirteen species of fresh water turtles were found in the Mogra River and its floodplain. Khan ${ }^{[27]}$ reported that Pangshura tecta are mainly distributed between the stretches of the Ganges River and the Brahmaputra River. Bengal Eyed turtle, Morenia petersi was found in the rivers and its flood plains wetland. Das ${ }^{[28]}$ mentioned its occurrence in Assam of India. Morenia petersi was regularly caught by fishermen and expert tribal hunters. Unfortunately, three important species of turtles became rare in their existence as per the catch data, within five years study period.

The population of bivalve, Lamellidens marginalis as found in the river and its flood plains has also been decreasing which is considered with the observation of Ali ${ }^{[29]}$ and Chakraborty ${ }^{[25]}$. During the study period, fresh water pearl bearing mussels (Bivalve, Lamellidens marginalis) were identified in the river. Shells of bivalve were utilized by rural people for production of lime which was utilized in aquaculture and agriculture land, and consumed with betel leaves and nuts.
The wildlife comprises amphibians (Bufo melanostictus, Rana tigerina, Rana limnocharis, Rana cyanophyctis and Salamandra salamandra etc.) aves (whistling duck, great crested grebe, great cormorant, red crested pochard, water cock, swamphen, great black headed gull, gray-headed fish eagle, curlew, spotted redshank etc.) and mammals (musk shrew, fishing cat, small Indian jackal, flying fox etc.) were previously reported by Chakraborty et al. ${ }^{[26]}$.

The study clearly indicates that the aquatic lives of the river were subjected to over exploitation resulting in gradual decline in their catch. The stock of aquatic animals is reducing due to pollution and destructive fishing practices ${ }^{[30,31,11]}$. Indiscriminate killing of fish occurred due to the use of pesticides in improper doses ${ }^{[6]}$, use of forbidden chemicals, and aerial spray of chemicals as used in paddy field which was very much similar to the observation of Chakraborty ${ }^{[31]}$ and Mazid ${ }^{[32]}$. Intervention to control floods, adoption of new agricultural technologies and construction of road networks altered the ecology of rivers and its flood plains significantly which supported the views of Khan ${ }^{[33]}$ and Ali ${ }^{[29]}$. Decreased stock of the wild brood fishes in their breeding ground also resulted in a reduction of biodiversity as noted by Nishat ${ }^{[34]}$, Zaman ${ }^{[35]}$ and Chakraborty ${ }^{[36]}$.

\section{Conclusions}

To save the stock of aquatic species in the river, a team of local management committee like Hilsa fisheries management technology is needed to develop a working frame-work. The deeper area of the river must be declared as a sanctuary to protect the aquatic lives, stricken enforcement of fish Act-1950 in the river, ensured stopping unplanned construction of flood control embankments, drainage system and sluice gates, conversion of inundated land to cropland (reducing water area); and controlling use of pesticides and agrochemicals in the floodplains of the river can save the ecosystems. The sustained produc- 
tion level from the river will also ensure livelihood of the fishers.

Acknowledgement The authors are greatfully acknowledged to Department of Fisheries, Bangladesh for kind suport and cooperation.

\section{References}

[1] Verma A. K. (2018), Ecological Balance: An Indispensable Need for Human Survival. Journal of Experimental Zoology, India. 21 (1), 407-409.

[2] Verma A. K. and Prakash S. (2020), Status of Animal Phyla in different Kingdom Systems of Biological Classification. International Journal of Biological Innovations. 2 (2): 149154. https://doi.org/ 10.46505/ IJBI.2020.2211.

[3] Noss R. F. (1990), A regional landscape approach to maintain diversity. Bioscience. 33, 700-706.

[4] Cairns M. A. and Lackey R.T. (1992), Biodiversity and management of natural resources: The issues Fisheries. 17(3), 6-10.

[5] Allendrof F. W. (1988), Conservation biology of fishes. Conservation Biology 2: 145-148.

[6] Prakash S. and Verma A. K. (2020). Effect of Organophosphorus Pesticides on Biomolecules of Fresh Water Fish, Heteropneustes fossilis (Bloch). Indian Journal of Biology. 7(2), 65-69.

[7] Disaster E. (1990),Floodplain protection in Central Europe. World Wildlife Found (WWF) Institute of Floodplains Ecology Visiting Card 31/90, Germany.

[8] Alam G.N. (1996), Floodplain fisheries development in Bangladesh. Fisheries News Bangladesh Fisheries Research Institute 4(1), 3-4.

[9] Shannon, C. E. (1948), A mathematical theory of communication. Bell System Tec. J.,27, 379-656.

[10] Zar, J. H. (1984), Biostatistics. Prentice-Hall, Inc. Englewood Cliffs, New Jersey, USA. 718.

[11] Chakraborty B. K., Bhattacharjee, S. and Muniya, S. (2021), A Study of aquatic biodiversity of Shuthi-Shaiduli river of Bangladesh. International Journal of Biological Innovations. 3(1), 58-67.

[12] Mathew, P. W. (1975), Limnology and productivity of Govindgarh Lake, Maddya Prodesh, India. Journal of Inland Fisheries Society, India 11, 16-24.

[13] Rahman, M. S. (1992), Water quality management in aquaculture. BARC Prokashana, 66, Mohakhali, Dhaka 1212, Bangladesh 84 pp.

[14] APHA (1998), Standard methods for the examination of water and wastewater, $20^{\text {th }}$ edition, American Public Health Association, Washington, DC.

[15] Boyd, C. E. (1982), Water Quality Management for Pond Fish Culture. Elsevier, The Netherlands. 318 pp.
[16] Clesceri L.S., Greenberg A.E. and Trussell R. R. (1989), Standard methods of the examination of water and wastewater $\left(17^{\text {th }}\right.$ ed.). American Public Health Association, American Water Works Association and Water Pollution Control Federation, 1015 Washington D. C., USA 20036, 10-203.

[17] Haroon A. K. Y., Halder G.C. Rahman S.L. Razzaque M.A. Alam M. and Amin S. M. N. (2002), Sylhet-mymensingh basin fish stock assessment. Final Report. Bangladesh Fisheries Research Institute, Reverine Station, Chandpur, Bangladesh 81 pp.

[18] Sugunan, V.V. and B.K. Bhattacharya. (2000), Ecology and fisheries of beels in Assam. Bull.No.104, CIFRI, Barrackpore-743101, West Bengal 66 pp.

[19] Chakraborty B. K. and Mirza M. J. A. (2010), Aquatic biodiversity of Someswari River in Bangladesh. Asian Fisheries Science 23, 174-193.

[20] Chakraborty B. K. and Mirza M. J. A. (2007), Aquatic biodiversity of Gharia Beel of Northern Bangladesh. J. Crop and Weed, India 3, 23-34.

[21] Moyle P. L. and Leidy R.A. (1992), Loss of biodiversity in aquatic ecosystem: evidence from fish fauna. In: P.L. Fielder and H.L. Jani (eds). Conservation of Biology: the theory and practice of nature conservation, preservation and management. Chapman and Hall, New York, USA. 562 pp.

[22] IUCN. (2000), Red book of threatened fishes of Bangladesh. IUCN- The World Conservation Union xii+116 pp.

[23] IUCN, Bangladesh. (1998), List of threatened animals of Bangladesh. Paper presented in the Special Workshop on Bangladesh Red Book of Threatened Animals, 22 February 1998, Dhaka, Bangladesh. 13 pp.

[24] Sarker, S. U. (1993), Faunal diversity and their conservation in freshwater wetlands. In Freshwater wetlands in Bangladesh issues and approaches for management. IUCN, The world Conservation Union, pp.105-122.

[25] Chakraborty B. K. (2009), Aquatic biodiversity of Someswari and Nethai River and Gharia and Nidaya Beel of Northern Bangladesh. In: Takumi K. Nakamura (ed.). Aquaculture Research Progress, Nova Science Publishers, New York, USA. 3231-3268p.

[26] Chakraborty B. K., Shahroz M. H., Bhuiyan A. B., Bhattacharjee S. and Chattoraj S. (2019), Status of Indian major carps spawns in the Halda River along with marketing and economic condition of the Fishers and related collectors. International Journal of Biological Innovations. 1 (2):40-50. https://doi. org/10.46505/IJBI.2019.1202.

[27] Khan, M.A.R. (1982), Chelonians of Bangladesh and 
their conservation. Journal of Bombay Natural History Society 79 (i), 110-116.

[28] Das, I. (1991), Color guide to the turtles and tortoises of the Indian sub continent. R \& A publishing Ltd.

[29] Ali, M. Y. (1991), Towards sustainable development: fisheries resources of Bangladesh International union for conservation of nature and natural resources. The World Conservation Union. National Conservation Strategy of Bangladesh and Bangladesh Agricultural Research Council. 96 pp.

[30] Mazid M. A. and Hossain, M. S. (1995), Development of fisheries resources in floodplains. FRI publication No. 12. Fisheries Research Institute, Mymensingh, Bangladesh.

[31] Chakraborty B. K. (2011), Present Status of Biodiversity in Bogajan Beel in Northern Bangladesh $J$. Fish. Soc. Taiwan 38(4), 277-300.

[32] Mazid M. A. (2002), Development of fisheries in Bangladesh: plans and strategic for income generation and poverty alleviation. Fisheries Research Institute, Mymensingh, Bangladesh. pp. 78-79.

[33] Khan H. R. (1993), Water development activities and their impacts on wetlands. P. 23-32. In: A. Nisat, Z. Hossain, M. K. Roy, and Ansarul Karim (eds.). Freshwater wetlands in Bangladesh: issues and approaches for management. IUCN, Gland Switzerland. xii $+283 p p$.

[34] Nishat A. (1993), Freshwater wetlands in Bangladesh: status and issues. P. 9-22 In: A. Nishat Z. Hossain, M. K. Roy, and Ansarul Karim (eds.). Freshwater wetlands in Bangladesh: Issues and Approaches for management. IUCN, Gland Switzerland. xii+283pp.

[35] Zaman, S. M. H. (1993), Agricultural development and sustainability of wetlands. pp.63-178 In: A. Nishat Z. Hossain, M.K. Roy, and A. Karim (eds.). Freshwater wetlands in Bangladesh: Issues and Approaches for management. IUCN, Gland Switzerland. xii+283p.

[36] Chakraborty, B. K. (2021). Induction of spawning and nursing pangas, Pangasianodon hypophthalmus (Sauvage, 1978) under hathery system. International Journal of Biological Innovations 3(2), 264-270. https://doi.org/10.46505/IJBI.2021.3203 